\title{
Dependence of the transference of a reduced eye on frequency of light*
}

\author{
T Evans and WF Harris
}

Department of Optometry, University of Johannesburg, PO Box 524, Auckland Park, 2006 South Africa

$<$ tevans@uj.ac.za>

$<$ wharris@uj.ac.za>

Received 7 July 2011; revised version accepted 25 October 2011

\begin{abstract}
In Gaussian optics the transference is a matrix that is a complete representation of the effects of the system on a ray traversing it. Almost all of the familiar optical properties of the system, such as refractive error and power of the system, can be calculated from the transference. Because of the central importance of the transference it is useful to have some idea of how it depends on the frequency of light. This paper examines the simplest model eye, the reduced eye. The dependence of the transference is calculated in terms of both frequency and
\end{abstract}

wavelength of light and both dependencies are displayed graphically. The principal matrix logarithms are also calculated and displayed graphically. Chromatic difference in refractive compensation, power and ametropia are obtained for the reduced eye from the transferences. ( $S$ Afr Optom 2011 70(4) 149-155)

Key Words: Transference, frequency, wavelength, Emsley's reduced eye, transformed transference, chromatic difference in: refractive compensation, power and ametropia.

\section{Introduction}

A transference is a matrix that represents the linear optical properties of an optical system, such as the eye. In Gaussian optics the transference is a complete representation of the effects of the system on a ray traversing it. Most of the optical properties of the system, such as refractive compensation ${ }^{1}$, back- and frontvertex power ${ }^{2}$, locations of the cardinal points ${ }^{3,4}$ and power of the system ${ }^{5}$ can be calculated from the system's transference. Because of the central importance of the transference it is useful to have some idea of how it depends on the frequency of the light traversing it. We take a look at the simplest model eye, the reduced eye.

The dependence of the fundamental first-order properties, calculated in terms of both frequency and wavelength of light, will be represented graphically across the visible light spectrum. Further, the dependence of the transformed transference will be represented graphically. Formulae for the calculation of chromatic difference in corneal-plane refractivecompensation of the reduced eye and chromatic difference in power and ametropia are derived.

\section{Emsley's reduced eye}

The advantage of the reduced eye (see Figure 1) is its simplicity. The reduced eye has a single stigmatic refracting surface of radius of curvature $r$ and a homogenous gap of length $z$. Emsley ${ }^{6}$ designed his reduced eye to match certain measurements of the Gullstrand-Emsley schematic eye. He placed specific emphasis on the power of $60 \mathrm{D}$ and chose the refractive index in the reduced eye to be the same as that of water, namely $4 / 3$. He took the index of air to be 1 . These numbers imply a radius of curvature $r$ of $50 / 9$ $\mathrm{mm}$ (or $1 / 180 \mathrm{~m}$ ) and a length $z$ of $200 / 9 \mathrm{~mm}$ (or $1 / 45$ 
m) ${ }^{6,7}$. In this paper we will make use of a reduced eye with the same $r$ and $z$ as Emsley's eye and with an index $n$ dependant on the frequency of light.

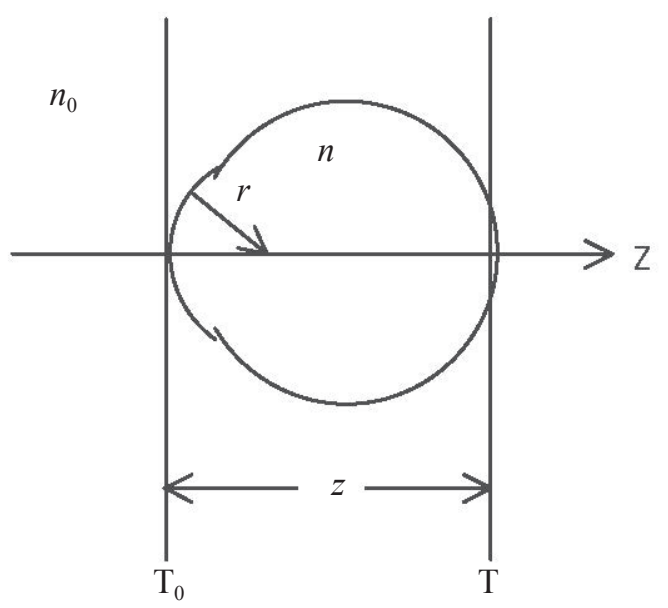

Figure 1. The reduced eye as a defined optical system. The length is $z$, the radius of curvature of the refracting surface is $r$, the refractive index outside the system is $n_{0}$ and inside the system is $n$. The optical system has a longitudinal axis, $Z$ and is bound by an entrance plane $T_{0}$ immediately in front of the refracting surface and an exit plane $\mathrm{T}$ immediately in front of the retinal surface.

\section{Linear optics}

An optical system is bound by an entrance plane $T_{0}$ and an exit plane $T$ and has a longitudinal axis $Z$. The transference $\mathbf{T}$ of a stigmatic, untilted, centred Gaussian optical system is represented by ${ }^{8}$

$$
\mathbf{T}=\left(\begin{array}{ll}
A & B \\
C & D
\end{array}\right)
$$

where $A$ the dilation, $B$ the disjugacy, $C$ the divergence and $D$ the divarication are the fundamental first-order optical properties of the system ${ }^{1,8,9}$. The power $F$ is given simply by 5,8

$$
F=-C \text {. }
$$

When the system is an eye the dilation $A$ can be considered to be the ametropia ${ }^{8}$ of the eye. In particular when the eye is emmetropic

$$
A=0 \text {. }
$$

The corneal-plane refractive compensation is given by 1,8

$$
F_{0}=B^{-1} A \text {. }
$$

The transferences of the two elementary optical systems ${ }^{10,11}$ are

$$
\mathbf{T}_{\zeta}=\left(\begin{array}{cc}
1 & \frac{z}{n} \\
0 & 1
\end{array}\right)
$$

for a homogenous gap and

$$
\mathbf{T}_{\mathrm{K}}=\left(\begin{array}{cc}
1 & 0 \\
-\frac{n-n_{0}}{r} & 1
\end{array}\right)
$$

for a refracting surface. To calculate the transference of the reduced eye one multiplies in reverse ${ }^{12}$ as follows

$\mathbf{T}=\mathbf{T}_{\xi} \mathbf{T}_{\mathrm{K}}$.

Substituting Equations 5 and 6 into Equation 7 and multiplying one obtains

$$
\mathbf{T}=\left(\begin{array}{cc}
1-\frac{z}{n}\left(\frac{n-n_{0}}{r}\right) & \frac{z}{n} \\
-\frac{n-n_{0}}{r} & 1
\end{array}\right) .
$$

Substituting the values for Emsley's reduced eye into Equation 8 one obtains

$$
\mathbf{T}=\left(\begin{array}{cc}
0 & \frac{50}{3} \mathrm{~mm} \\
-0.060 \mathrm{kD} & 1
\end{array}\right) .
$$

It is immediately apparent from Equations 3 and 9 that the eye is emmetropic and has a power of $60 \mathrm{D}$.

\section{Frequency or wavelength?}

The fundamental relationship between frequency $(v)$ and vacuum wavelength $(\lambda)$ is given by $\mathrm{c}_{0}=v \lambda$

where light traveling in a vacuum has a speed $\mathrm{c}_{0}=299$ $792458 \mathrm{~m} . \mathrm{s}^{-1}$ as defined by the 17th General Conference on Weights and Measures in November 1983. Pease and Barbeito ${ }^{13}$ look at the relationship between frequency and wavelength for a number of studies involving chromatic aberration and conclude that results using frequency or wavenumber (the inverse of wavelength) are "nearly perfectly linear" 13,14 in contrast to those using wavelength. They cite several reasons to support using frequency rather than wavelength (perhaps the most important being that frequency is independent of the medium whereas wavelength is not). These reasons make a compelling argument to study 
the dependence of the transference on the frequency of light rather than its dependence on wavelength. We will compare the dependence of the transference on both frequency and wavelength.

\section{Visible light colour spectrum}

The spectrum with wavelengths in vacuum is between 400 and $700 \mathrm{~nm}$ and represents the range over which human spectral sensitivity varies between 1 and $100 \% .^{14-16}$ This represents frequencies between 428.3 $\mathrm{THz}$ and $749.5 \mathrm{THz}$, approximately. Six coloured reference points are shown on each of the graphs below. The four colours red-orange, yellow, green and blue represent the peak vacuum wavelengths where each colour is considered "pure" 17 while the deep-red and violet-blue represent the spectral range end-points. The frequencies and wavelengths of the six colours are detailed in Table 1.

\section{Transference as a function of refractive index}

The transference of the reduced eye in Equation 8 shows that the variable affected by different frequencies of light is the refractive index $n$. Entering the radius of curvature and length of Emsley's reduced eye we obtain its transference as a function of the refractive indices:

$\mathbf{T}=\left(\begin{array}{cc}\frac{4 n_{0}}{n}-3 & \frac{200}{9 n} \mathrm{~mm} \\ -\frac{9}{50}\left(n-n_{0}\right) \mathrm{kD} & 1\end{array}\right)$.

\section{Refractive index of the Reduced Eye}

Thibos et al ${ }^{15}$ represent the refractive index of the reduced eye as a function of wavelength as follows

$n=a+\frac{b}{\lambda-c}$

where $\mathrm{a}=1.320535, \mathrm{~b}=4.685 \mathrm{~nm}$ and $\mathrm{c}=214.102 \mathrm{~nm}$. The formula is based on Cornu's formula for refractive index of water and constants were derived from clinical experimentation on real eyes. Using this formula, Thibos et al ${ }^{15}$ showed that the refractive index of the body of the reduced eye changes more rapidly with wavelength than a reduced eye filled with water. The predictions for longitudinal chromatic aberration using this formula more closely approximate experimental data than Emsley's reduced eye filled with water. The refractive indices for our six reference points were calculated using Equation 12 and are given in Table 1.

\section{Refractive index of air}

The refractive index of air differs only very slightly from that of a vacuum and for most optometric calculations one can put $n_{0}=1$. A number of equations, for example Cauchy's dispersion formula ${ }^{18}$ and Ciddor's equations ${ }^{19}$, are available for calculating the refractive index of air. Cauchy's formula is expressed in terms of wavelength whereas Ciddor's equations are expressed in terms of wavenumber. Cauchy's dispersion formula ${ }^{18}$ is

$$
\left(n_{0}-1\right) 10^{7}=p+\frac{q}{\lambda^{2}}+\frac{t}{\lambda^{4}}
$$

Table 1. The colours of the six reference points and their frequencies, vacuum wavelengths, refractive indices for the reduced eye, ametropias, powers for the reduced eye and refractive compensations are provided. The last row shows the chromatic difference across the spectrum 428.3 to $749.5 \mathrm{THz}$.

\begin{tabular}{lllllll}
\hline Colour & $\begin{array}{l}\text { Frequency } \\
\text { in THz }\end{array}$ & $\begin{array}{l}\text { Vacuum } \\
\text { wave- } \\
\text { length in } \\
\text { nm }\end{array}$ & $\begin{array}{l}\text { Refractive } \\
\text { index }\end{array}$ & Ametropia & $\begin{array}{l}\text { Power of } \\
\text { reduced eye } \\
\text { in dioptres }\end{array}$ & $\begin{array}{l}\text { Refractive } \\
\text { compensation } \\
\text { in dioptres }\end{array}$ \\
\hline Deep red & 428.27 & 700 & 1.3302 & 0.0071 & 59.4318 & 0.4261 \\
Red & 475.86 & 630 & 1.3318 & 0.0035 & 59.7240 & 0.2070 \\
Yellow & 516.88 & 580 & 1.3333 & 0.0000 & 60.0010 & 0.0008 \\
Green & 576.52 & 520 & 1.3359 & -0.0057 & 60.4531 & -0.3398 \\
Blue & 631.14 & 475 & 1.3385 & -0.0116 & 60.9286 & -0.6964 \\
Violet-blue & 749.48 & 400 & 1.3457 & -0.0277 & 62.2327 & -1.6745 \\
\hline Chromatic difference in: & & $\Delta n$ & $\Delta A$ & $\Delta F$ & $\Delta F_{0}$ \\
\hline
\end{tabular}


where $\mathrm{p}=2726.43, \mathrm{q}=12.288 \times 10^{6} \mathrm{~nm}^{2}$ and $\mathrm{t}=355.5 \times 10^{9}$ $\mathrm{nm}^{4}$ for dry air at temperature $15^{\circ} \mathrm{C}$ and pressure 101 $\mathrm{kPa}$.

When Cauchy's dispersion formula is used for $n_{0}$ one obtains results that differ insignificantly from those for $n_{0}=1$. In particular for dilation the difference is less than 0.00083 across the entire visible light spectrum. For divergence this difference is $0.05 \mathrm{D}$. This results in a very slight upward shift in the graphs for $A$ and $C$ in Figure 2, however, the curvatures are unaffected.

Calculation shows that the reduced eye is emmetropic at the frequency $517 \mathrm{THz}(580 \mathrm{~nm}$, yellow) when we use $n_{0}=1$ but is emmetropic at the frequency $526 \mathrm{THz}$ (570 nm, yellow-green) when the refractive index of air is calculated using Cauchy's formula.

\section{Graphical representation of the fundamental properties}

The properties are calculated for frequencies according to Equation 11 with $n_{0}=1$ and $n$ given by Equation 12. The results of the calculations of each of the fundamental first-order optical properties are given in the accompanying graphs. Figure 2 represents each of the fundamental properties as functions of frequency of light and Figure 3 as functions of wavelength. The six coloured reference points are shown by means of coloured diamonds. The small black dots on Figure 2 represent 10 equally spaced intervals of frequency of approximately $32.1 \mathrm{THz}$ and the crosses on Figure 3 represent 10 equally spaced intervals of $30 \mathrm{~nm}$ wavelength.

We note that in Figure 2 the dots are more evenly spaced than the crosses in Figure 3. The dashed line represents the slope of the curve calculated using the least squares method. Each of $A, B$ and $C$ present as curves in both sets of graphs. $D$ is a straight line at 1 as required by Equation 11. The curves are closer to straight lines in Figure 2 than in Figure 3; this provides some justification for preferring to think in terms of frequency rather than wavelength of light.

The transferences at the extremes of the visible spectrum $(428.3 \mathrm{THz}$ or $700 \mathrm{~nm}$ and $749.5 \mathrm{THz}$ or $400 \mathrm{~nm})$, are

$$
\begin{aligned}
& \mathbf{T}_{428.3 \mathrm{THz}}=\left(\begin{array}{cc}
0.0071 & 16.7062 \mathrm{~mm} \\
-0.0594 \mathrm{kD} & 1
\end{array}\right) \\
& \mathbf{T}_{749.5 \mathrm{THz}}=\left(\begin{array}{cc}
-0.0277 & 16.5130 \mathrm{~mm} \\
-0.0622 \mathrm{kD} & 1
\end{array}\right) .
\end{aligned}
$$
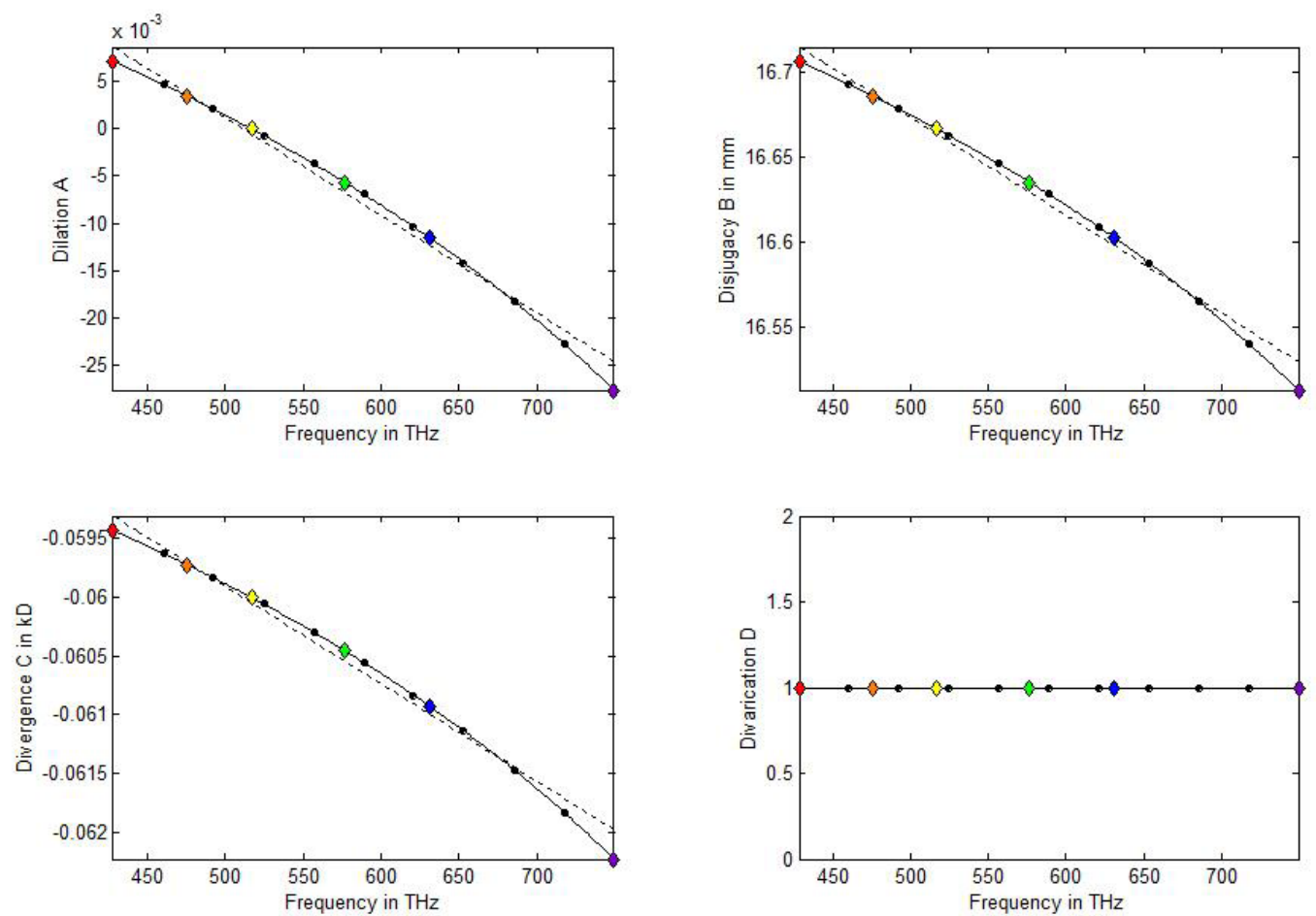

Figure 2. Sub-graphs A, B, C and D represent the four fundamental optical properties of the reduced eye as a function of frequency of light. The six coloured diamonds indicate six reference points as indicated in Table 1 . The small black dots represent 10 equal intervals of $32.1 \mathrm{THz}$ each. Each of $A, B$ and $C$ present as curves, while $D$ is a horizontal straight line at 1. 

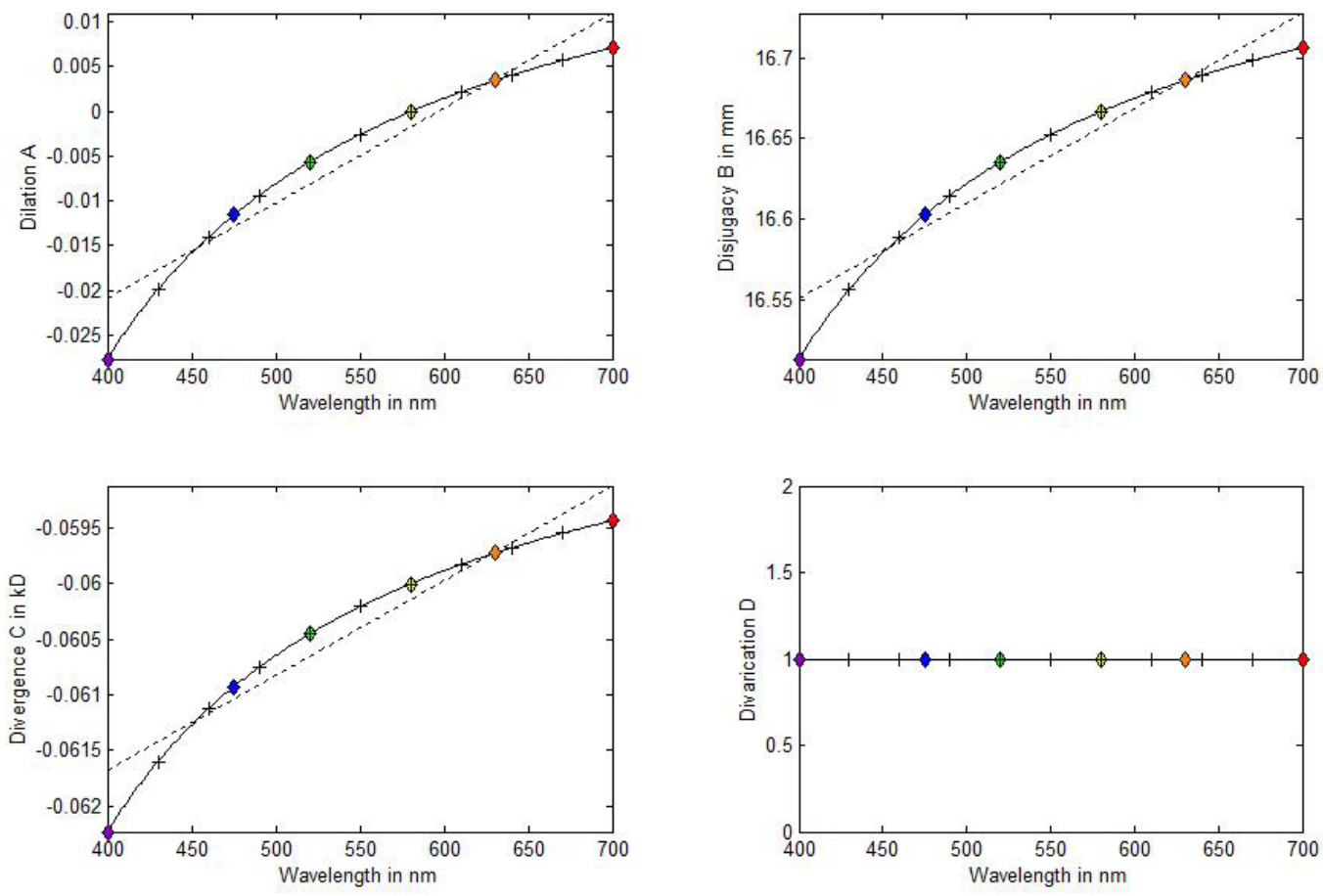

Figure 3. The four fundamental properties of the reduced eye as a function of wavelength. The black crosses represent 10 equal intervals of $30 \mathrm{~nm}$ each.

\section{The transformed transference}

By taking the principal matrix logarithm we convert each transference $T$ into a Hamiltonian matrix 8,20 . We represent the transformed matrix ${ }^{8}$ by $\hat{\mathbf{T}}$. Thus

$$
\hat{\mathbf{T}}=\log \mathbf{T} \text {. }
$$

In MATLAB the function used is logm. In terms of its entries we write the transformed transference ${ }^{8}$ as

$\hat{\mathbf{T}}=\left(\begin{array}{cc}\hat{A} & \hat{B} \\ \hat{C} & \hat{D}\end{array}\right)$.

Because $\hat{\mathbf{T}}$ is Hamiltonian ${ }^{20}$,

$\hat{A}=-\hat{D}$.

$\hat{\mathbf{T}}$ therefore has only three independent entries. This creates a 3-dimensional vector space which can be plotted on a 3-dimensional graph as done in Figure 4. In the figure $\hat{A}, \hat{B}$ and $\hat{C}$ are along three orthogonal axes, where $\hat{A}$ (and $\hat{D}$ ) are unitless, $\hat{B}$ is in millimetres and $\hat{C}$ is in kilodioptres. The result is close to a straight line. The small black dots represent 20 equally spaced intervals of frequency of $16.1 \mathrm{THz}$.

Transforming Equations 14 and 15, we find

$$
\begin{gathered}
\hat{\mathbf{T}}_{428.3 \mathrm{THz}}=\left(\begin{array}{cc}
-0.5994 & 20.1699 \mathrm{~mm} \\
-0.0718 \mathrm{kD} & 0.5994
\end{array}\right) \\
\hat{\mathbf{T}}_{749.5 \mathrm{THz}}=\left(\begin{array}{cc}
-0.6251 & 20.0888 \mathrm{~mm} \\
-0.0757 \mathrm{kD} & 0.06251
\end{array}\right) .
\end{gathered}
$$

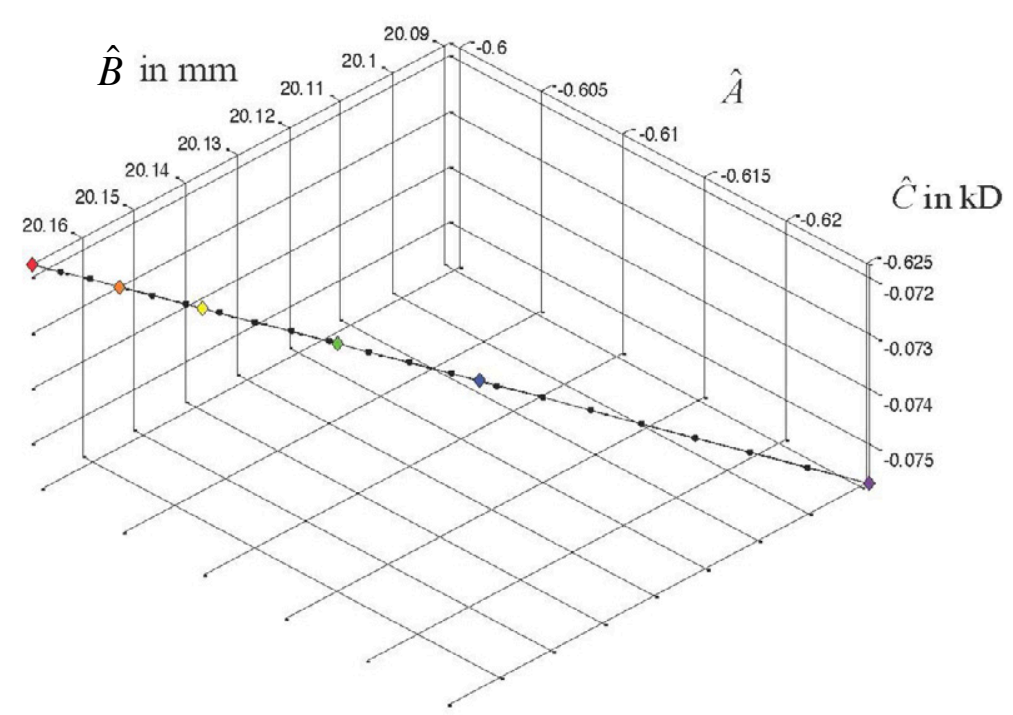

Figure 4. The transformed transference of the reduced eye in Hamiltonian space. The three axes represent $\hat{A}, \hat{B}$ and $\hat{C}$. The black dots represent frequencies spaced at intervals of 16.06 THz. The six diamonds represent the six coloured reference points. (The azimuth of $45^{\circ}$ and elevation of $125^{\circ}$ were chosen to exaggerate any possible curvature.) 


\section{Some Derived Properties}

We obtain here some derived properties of the reduced eye that are directly and simply obtainable from the transference. Using Equation 2 we calculate the power $F$ of the reduced eye for a particular frequency of light and using Equation 4 we calculate the corneal-plane refractive compensation $F_{0}$ for the frequency. We read the ametropia $A$ directly from the transference for the chosen frequency of light. Of greater interest is the chromatic difference in refractive compensation $\Delta F_{0}$ (known by various terms including longitudinal chromatic aberration ${ }^{21}$, chromatic difference of refractive error ${ }^{21}$, chromatic difference in refraction ${ }^{14}$, and axial chromatic aberration $^{21}$ ), chromatic difference in power (also known as chromatic difference of equivalent power ${ }^{14}$ ) and chromatic difference in ametropia across a specified spectrum of visible light to be studied.

Each of these derived properties in chromatic difference between two frequencies or wavelengths can be calculated in two ways: either directly from the two transferences or using the equations below. Equations 21, 23, 24 and 26 are general equations while Equations 22, 25 and 27 apply to the reduced eye.

To calculate the chromatic difference in cornealplane refractive compensation across a specified spectrum we take values from the two transferences (Equation 1) and substitute them into Equation 4 as follows:

$\Delta F_{0}=B_{2}^{-1} A_{2}-B_{1}^{-1} A_{1}$.

Now substituting from Equation 8 and also substituting the values for $z$ and $r$ for the reduced eye, we find $\Delta F_{0}=(-135 \mathrm{D}) \Delta n$

where $\Delta \mathrm{n}$ simplifies to

$$
\Delta n=b\left(\frac{1}{\lambda_{2}-c}-\frac{1}{\lambda_{1}-c}\right)
$$

and $b$ and $c$ are the same constants as for Equation 12 .

To calculate chromatic difference in power we similarly take values from the transferences and substitute them into Equation 2 as follows

$\Delta F=-\left(C_{2}-C_{1}\right)$

and substituting from Equation 8 and then substituting the value for $r$ for the reduced eye we find $\Delta F=(180 \mathrm{D}) \Delta n$.
The chromatic difference in ametropia is derived directly from the transferences and therefore

$\Delta A=A_{2}-A_{1}$.

Substituting values for $z$ and $r$ we obtain

$\Delta A=4\left(\frac{1}{n_{2}}-\frac{1}{n_{1}}\right)$

where $n_{1}$ and $n_{2}$ are calculated from Equation 12.

The chromatic difference in corneal-plane refractive compensation of the reduced eye was calculated across the visible light spectrum 428.3 to $749.5 \mathrm{THz}$ and is $-2.1006 \mathrm{D}$ (see Table 1). The chromatic difference in power is $2.8 \mathrm{D}$. The chromatic difference in ametropia is -0.0348 .

\section{Conclusion}

The transference of the reduced eye depends on the frequency of light. The accuracy of the calculations depends on the formula used to calculate the refractive index as a function of either frequency of light or wavelength. In this article we have used Equation 12.

Results are displayed graphically for the transferences both as a function of frequency and as a function of wavelength. Divarication $D$ is constant while ametropia $A$, disjugacy $B$ and divergence $C$ exhibit curved lines. The graph for the transformed transference in Hamiltonian space is approximately a straight line.

Chromatic difference in refractive compensation, chromatic difference in power and chromatic difference in ametropia are calculated directly from the transference for the reduced eye.

\section{Acknowledgements}

We thank RD van Gool for continued discussions. WF Harris acknowledges support from the National Research Foundation. All calculations and graphs were done using MATLAB.

\section{References}

1. Harris WF. Magnification, blur, and ray state at the retina for the general eye with and without a general optical instrument in front of it. 1. Distant objects. Optom Vis Sci 2001 78 888-900.

2. Harris WF. Back- and front-vertex powers of astigmatic systems. Optom Vis Sci 201087 70-72.

3. Harris WF. Graphical construction of cardinal points from the transference. S Afr Optom 2011 70 3-13. 
4. Harris WF. Pascal's ring, cardinal points, and refractive compensation. Vision Res 201151 1679-1685.

5. Harris WF. Dioptric power: its nature and its representation in three- and four-dimensional space. Optom Vis Sci 1997 74 349-366.

6. Emsley HH. Visual Optics. London: Hatton Press Ltd, 1950 pp 525-527, 543-544.

7. Bennett AG, Rabbetts RB. Clinical Visual Optics. London: Butterworth. 1984; p 18.

8. Harris WF. The log-transference and an average Gaussian eye. S Afr Optom 200564 84-88.

9. Harris WF. The four fundamental properties of Gaussian optical systems including the eye. S Afr Optom 199958 69-79.

10. Harris WF. General approach to the sensitivity of the optics of an eye to change in elementary parameters with application to the Gaussian optics of a reduced eye. S Afr Optom 200968 166-174.

11. Harris WF. Symplecticity and relationships among the fundamental properties in linear optics. S Afr Optom 201069 $3-13$.

12. Harris WF. Stigmatic optical systems. Optom Vis Sci 2004 81 947-952.

13. Pease PL, Barbeito R. Axial chromatic aberration of the human eye: frequency or wavelength? Ophthal Physiol Opt $19899215-217$.

14. Rabbetts RB. Bennett and Rabbetts' Clinical Visual Optics, 4th Ed. Edinburgh: Butterworth Heineman Elsevier, 2007 pp 287-293.

15. Thibos LN, Ye M, Zhang X, Bradley A. The chromatic eye: a new reduced-eye model of ocular chromatic aberration in humans. Appl Opt 199231 3594-3600.

16. Le Grand Y. Light, Colour and Vision. Chapman and Hall, London; 1957 pp 72-73.

17. Meyer-Arendt JR. Introduction to Classical and Modern Optics. New Jersey: Prentice Hall, 1995 p 5.

18. Hodgson CD. Handbook of Chemistry and Physics. Chemical Rubber Publishing Co, Cleveland, 1959 p 2943.

19. Ciddor PE. Refractive index of air: new equations for the visible and near infrared. Appl Opt 199635 1566-1573.

20. Harris WF. Quantitative analysis of transformed ray transferences of optical systems in space of augmented Hamiltonian matrices. S Afr Optom 200766 62-67.

21. Thibos LN, Bradley A, Zhang X. Effect of ocular chromatic aberration on monocular visual performance. Optom Vis Sci $199168599-607$. 